POS PROCEEDINGS

\title{
New XMM-Newton observations of type 1 INTEGRAL AGN
}

\author{
Francesca Panessa*, Angela Bazzano, Alessandra De Rosa, Pietro Ubertini \\ INAF - Istituto di Astrofisica Spaziale e Fisica Cosmica - Roma \\ E-mail: francesca.panessa@iasf-roma.inaf.it
}

\section{Loredana Bassani, Angela Malizia}

INAF - Istituto di Astrofisica Spaziale e Fisica Cosmica - Bologna

\section{Manuela Molina}

INAF - Istituto di Astrofisica Spaziale e Fisica Cosmica - Milano

\section{Antony Bird, Antony Dean}

School of Physics and Astronomy, University of Southampton, Southampton

\begin{abstract}
The INTEGRAL/IBIS is surveying a large fraction of the sky above $20 \mathrm{keV}$ with a sensitivity larger than a few mCrab, discovering a number of new extra-galactic sources many of which now identified as nearby AGN. We have selected a complete sample of INTEGRAL hard X-ray AGN and we have combined INTEGRAL data with 2-10 keV information to probe the broad-band spectral properties of different classes of AGN. We will present new XMM-Newton observations of type 1 Seyfert galaxies and Narrow Line Seyfert 1 galaxies. By combining INTEGRAL data with XMM-Newton measurements we are now able to probe the average properties of type 1 hard $\mathrm{X}$-ray selected AGN spectra, in particular power law index, high energy cut-off and reprocessing features.
\end{abstract}

The Extreme sky: Sampling the Universe above $10 \mathrm{keV}$ - extremesky2009,

October 13-17, 2009

Otranto (Lecce) Italy

\footnotetext{
${ }^{*}$ Speaker.
} 


\section{Introduction}

The INTEGRAL/IBIS (e.g., the 4th IBIS catalogue, Bird et al. 2010) and Swift/BAT (e.g., the 22-months catalogue, Tueller et al. 2009) observatories are providing the best yet surveys of the hard X-ray/soft gamma-ray sky above $20 \mathrm{keV}$ with a sensitivity larger than a few mCrab and a point source location accuracy of the order of 1-3 arcmins depending on the source strength. This has consequently increased the AGN statistics and demography above $20 \mathrm{keV}$, providing an invaluable list of bright hard-X-ray AGN to perform broad-band X-ray spectroscopy.

Indeed, information on the broad-band spectra of these powerful sources is crucial for a proper determination of the AGN primary continuum, its high energy cut-off and reprocessing features. These are key parameters for the study of the physical characteristics of the Comptonizing region around the central nucleus. For instance, the current models ascribe the power law continuum shape as due to the inverse Compton scattering of soft photons in a corona of 'hot electrons' (e.g. Maraschi \& Haardt 1997; Zdziarski et al. 1998).

The average power-law photon index (and its spread in values) and the reflection component are other fundamental input parameters when considering the AGN contribution to the current XRB models (Gandhi et al. 2007; Gilli, Comastri \& Hasinger 2007).

Although broad-band measurements of AGN have been made in the past, mainly with the BeppoSAX satellite (e.g. Perola et al. 2002; Dadina 2007), a proper determination of the AGN continuum and its reprocessing features on broad band basis is still poorly explored.

\section{The sample}

From the third INTEGRAL/IBIS survey (Bird et al 2007), we have extracted a complete sample of AGN. By applying the Ve/Va relationship, we have obtained a complete sample of 88 AGN selected in the 20-40 keV energy band detected at $>5 \sigma$ confidence level (Malizia et al. 2009). All the AGN in the sample have an accurate optical classification (Masetti et al. 2009 and references therein). The sample consists of 46 Seyfert 1 galaxies (including 5 Narrow Line Seyfert 1, NLS1), 22 Seyfert 2 and 9 Blazars.

We have already analysed the broad-band spectral properties of non-complete samples of hard X-ray selected AGN (De Rosa et al. 2008, Panessa et al 2008, Molina et al. 2008, Malizia et al. 2007) and recently Molina et al. (2009) presented the study of the 36 brightest type 1 AGN in the Malizia et al. (2009) complete sample. In all these works we have combined archival data below $10 \mathrm{keV}$ (XMM-Newton, Chandra, Swift/XRT, ASCA, etc.) to characterize the broad-band spectral parameters. In particular, the combination of the INTEGRAL data with the XMM-Newton spectra provides a very good statistical quality data over the $0.1-150 \mathrm{keV}$ energy band.

Here, we present a preliminary broad-band spectra analysis of a sample of 7 Seyfert 1 galaxies and 6 NLS1 ( 5 from the complete sample and one detected by Swift/BAT, i.e., Swift J0923.7+2255) having new XMM-Newton data sets (XMM-Newton AO-8). These data have been combined with, whenever available, INTEGRAL/IBIS (from the 3th IBIS catalogue, Bird et al. 2007) and Swift/BAT spectra (from the BAT 22-Month Source Catalog, Tueller et al. 2009). The cross calibration constants between the three instruments have been fixed to 1 (see Molina et al. 2009 for a detailed discussion on the XMM/IBIS/BAT cross calibration constants). To characterize the 

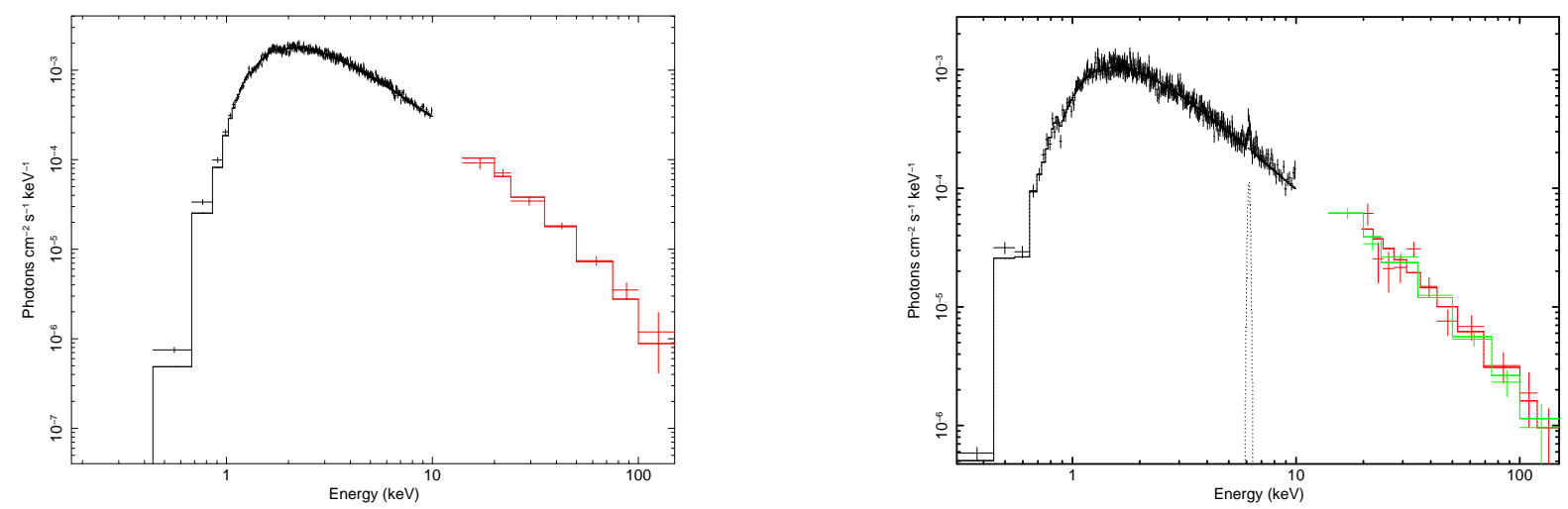

Figure 1: XMM-Newton (black) and INTEGRAL/IBIS (red) broad-band spectrum of GRS 1734-2212 (left panel). XMM-Newton (black), INTEGRAL/IBIS (red) and Swift/BAT (green) broad-band spectrum of QSO B0241+62 (right panel).
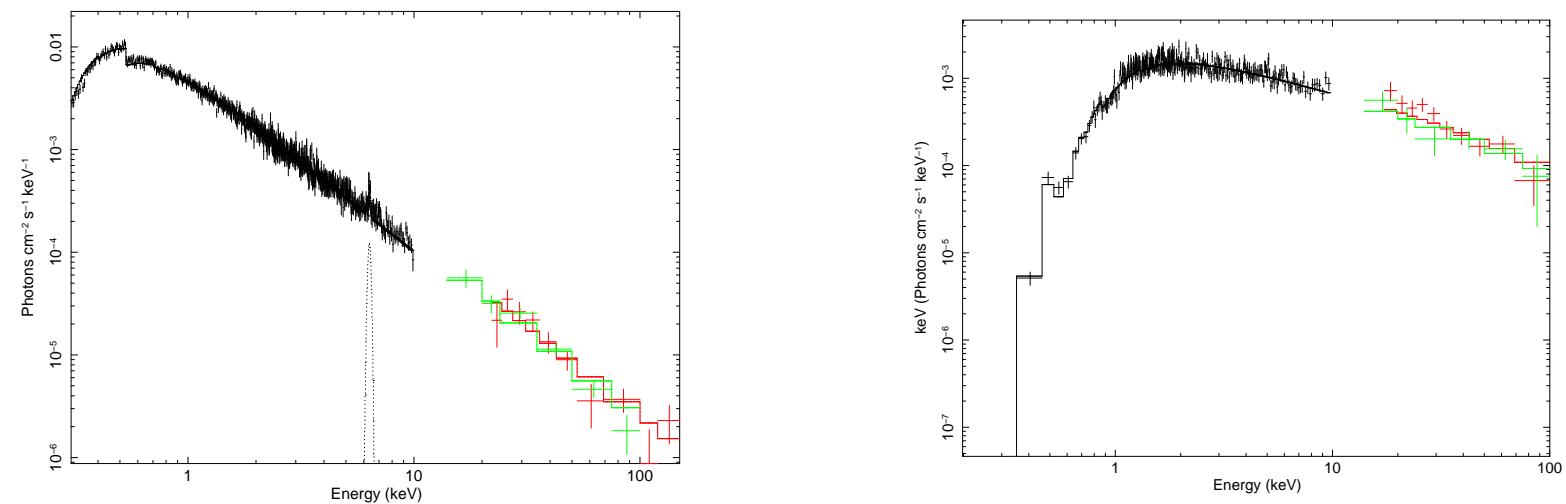

Figure 2: XMM-Newton (black), INTEGRAL/IBIS (red) and Swift/BAT (green) broad-band spectrum of NGC 6814 (left panel) and 2E 1853.7+1534 (right panel).

spectrum in the $0.1-150 \mathrm{keV}$ energy range, we have adopted the pexrav model in XSPEC, i.e. an exponentially cut-off power law reflected from neutral material, where the reflection component is described by the parameter $\mathrm{R}=\Omega / 2 \pi$, and the inclination angle has been fixed to $30^{\circ}$, i.e. a nearly face-on geometry as expected for type 1 sources. A narrow Gaussian component has also been included to take into account the presence of the $\mathrm{Fe} \mathrm{K} \alpha$ line at $6.4 \mathrm{keV}$. Galactic and intrinsic absorption are as well added to the model.

\section{Type 1 Seyfert galaxies}

In Molina et al. (2009), the study of the 36 brightest type 1 AGN of the IBIS AGN complete sample allowed improved estimates of all relevant parameters: the photon index distribution peaks at 1.7 (with a dispersion of 0.2), the high energy cut-off ( $\mathrm{see}_{\mathrm{E}} \mathrm{E}_{\text {cut }- \text { off }}$ ranges between $50-150 \mathrm{keV}$ ), the $\mathrm{FeK} \alpha$ narrow line equivalent width ranges between $70-80 \mathrm{eV}$ and the mean value for the reflection parameter $\mathrm{R}$ is 1 , but with a tail extending towards high reflection values. Finally, complex absorption in the form of 2-3 layers of material partially covering the nucleus was observed in $24 \%$ of the sample. In this previous analysis, XMM-Newton data were used for 24 objects, while the remaining sources only had archival observations from Swift-XRT/ASCA/Chandra. Here we 

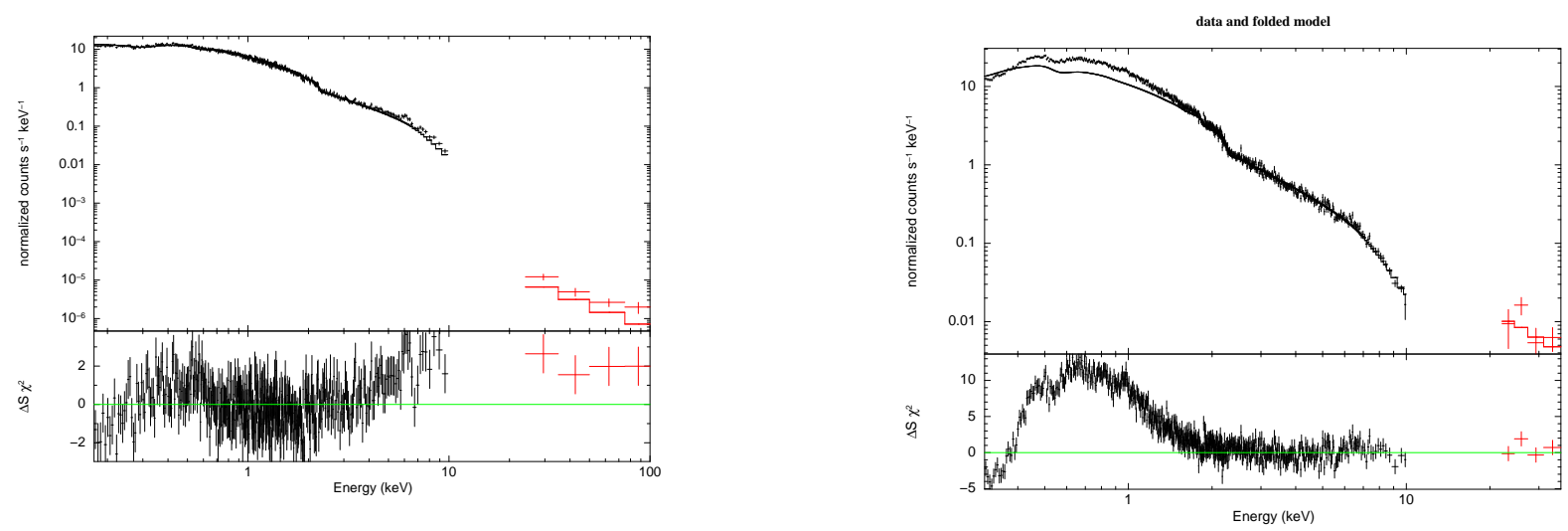

Figure 3: XMM-Newton (black) and Swift/BAT (red) broad-band spectrum of Swift J0923.7+2255 (left panel). XMM-Newton (black) and INTEGRAL/IBIS (red) broad-band spectrum of IGR J19378-0617 (right panel).
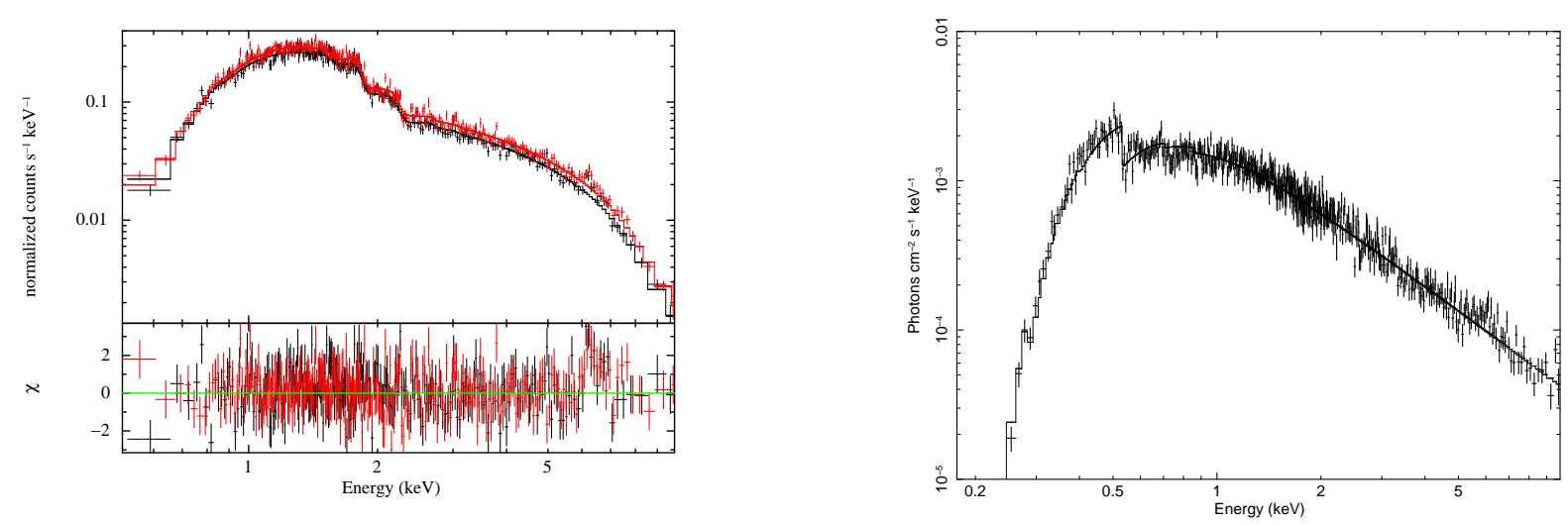

Figure 4: XMM-Newton (black), INTEGRAL/IBIS (red) and Swift/BAT (green) broad-band spectrum of NGC 6814 (left panel) and 2E 1853.7+1534 (right panel).

present new XMM-Newton data now available for 7 Seyfert 1 galaxies belonging to the IBIS AGN complete sample (Malizia et al. 2009, Molina et al. 2009).

In figure 1 and 2 we show the broad-band XMM-Newton and INTEGRAL/IBIS spectra of four out of the seven Seyfert 1 galaxies, in the case of QSO B0241+62, NGC 6814 and 2E 1853.7+1534 the Swift/BAT spectra above $20 \mathrm{keV}$ are also used. Interestingly, the photon index distribution of these sources peaks at Gamma 1.6, flatter than the canonical AGN index of 1.8-1.9 (Piconcelli et al. 2005). We were able to constrain the high energy cut-off in four out of seven sources, with values ranging from $50 \mathrm{keV}$ to $130 \mathrm{keV}$, consistent with Molina et al. (2009). In six out of seven sources a FeK $\alpha$ narrow line is detected, although in two cases the spectra is noisy around the 6-7 $\mathrm{keV}$ energy band. A significant reflection component is required in three source of the sample. A complete and detailed analysis of the data sets will be presented in Molina et al. in preparation.

\section{Narrow Line Seyfert 1 galaxies}

Among the important discoveries of INTEGRAL, the detection of NLS1 above $20 \mathrm{keV}$ is certainly one of the most notable. NLS1 galaxies are a very peculiar sub-class of AGN displaying 
high spectral complexity, therefore the study of their broad band spectra can allow the disentangling of the different proposed theoretical models. Malizia et al. (2008) reported the study of the broad band properties of a small sample of NLS1 by combining the IBIS spectra with the Swift/XRT data below $10 \mathrm{keV}$. The data showed that the photon index in this class of sources is steeper than typically observed in Seyfert 1 galaxies, as already found in previous studies. They also measured low values of the high energy cut-off $\left(\mathrm{E}_{c}<60 \mathrm{keV}\right)$.

Our preliminary analysis of the new XMM-Newton data sets reveal high complexity in the spectrum of the observed NLS1 (see figure 3 and 4). First of all, we confirm the evidence of a steep photon index (Gamma $\sim 1.9-2)$. Also in our work, where Seyfert 1 galaxies and NLS1 have been selected in the same manner (in the $20-40 \mathrm{keV}$ energy range), a different photon index distribution is found, with NLS1 having steeper Gamma. Malizia et al. (2008) suggested that this steepening is a consequence of the low value of the energy cut-off measured which flatten the spectrum. However, we were able to obtain a lower limit on the high energy cut-off in only two sources and the values found are consistent with those in Malizia et al. (2008). As in Malizia et al. (2008), the reflection component is not strongly required, although in at least two sources, there is a clear excess emission above $20 \mathrm{keV}$ with respect to the XMM-Newton data.

An FeK $\alpha$ narrow line is always detected, with two sources showing strong evidence of a broad Gaussian component. In one case, SWIFT J2127.4+5654, Suzaku data have confirmed the presence of a low energy cut-off and have allowed the detection of a broad relativistic iron emission line, from its profile we were able to estimate the black hole spin (Miniutti et al. 2009). A strong and complex soft component is detected in four sources, suggesting that the non detection of this component in the XRT data in Malizia et al. (2008) is due to the lower sensitivity of the instrument. A detailed analysis and discussion of the broad band properties of this representative hard X-ray selected sample of NLS1 will be presented in a future work (Panessa et al. in preparation).

\section{References}

[1] Bird, A. J., et al. 2010, The Astrophysical Journal Supplement, 186, 1

[2] Bird, A. J., et al. 2007, The Astrophysical Journal Supplement, 170, 175

[3] Dadina M., 2007, Astronomy and Astrophysics, 461, 1209

[4] De Rosa, A. et al. 2008 Astronomy and Astrophysics, 483, 749

[5] Gandhi, P., et al. 2007, MNRAS, 382, 1005

[6] Gilli, R., Comastri, A., \& Hasinger, G. 2007, Astronomy and Astrophysics, 463, 79

[7] Malizia, A., et al. 2009, MNRAS, 399, 944

[8] Malizia, A., et al. 2008, MNRAS, 389, 1360

[9] Malizia, A., et al. 2007, The Astrophysical Journal, 668, 81

[10] Maraschi L., Haardt F., 1997, in Wickramasinghe D. T., Bicknell G. V., Ferrario L., eds, ASP Conf. Ser. Vol. 121, Accretion Phenomena and Related Outflows, IAU Coll. 163. Astron. Soc. Pac., San Francisco, p. 101

[11] Masetti, N., et al. 2009, Astronomy and Astrophysics, 495, 121 
[12] Miniutti, G., et al. 2009, MNRAS, 398, 255

[13] Molina, M., et al. 2009, MNRAS, 399, 1293

[14] Molina, M. et al. 2008, MNRAS, 390, 1217M

[15] Panessa, F.et al. 2008, Astronomy and Astrophysics, 483, 151

[16] Perola G. C.,et al. 2002, Astronomy and Astrophysics, 389, 802

[17] Piconcelli E.,et al. 2005, Astronomy and Astrophysics, 432, 15

[18] Tueller, J., et al. 2009, arXiv:0903.3037

[19] Zdziarski A. A., 1998, MNRAS, 301, 435 